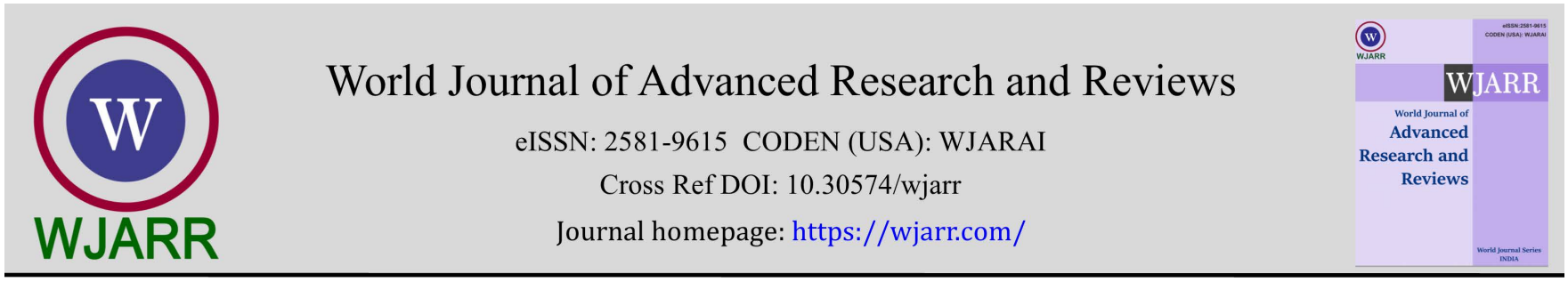

(CASE REPORT)

Check for updates

\title{
Alagille Syndrome: A case report of a rare oral and multisystem manifestation
}

\author{
Adidémè Monique EZIN * \\ University of Mohammed V, Faculty of dentistry, Departement of Pediatric Dentistry, Rabat-MOROCCO
}

World Journal of Advanced Research and Reviews, 2022, 13(01), 317-321

Publication history: Received on 05 December 2021; revised on 07 January 2022; accepted on 09 January 2022

Article DOI: https://doi.org/10.30574/wjarr.2022.13.1.0012

\begin{abstract}
Alagille syndrome is an inherited multisystem disorder of autosomal dominant transmission. Its prevalence is estimated at 1 per 70,000 to 100,000 live births. We report the case of a young patient suffering from Alagille syndrome who consulted the center of diagnosis and dental treatment of Rabat - MOROCCO (CCTD). The general manifestations are facial dysmorphia, hepatic, cardiac, and ocular disorders. Hepatic cholestasis causes oral repercussions such as a yellow oral mucosa, hypomineralization of the teeth, and a high tendency to dental caries.

The management of such a patient requires the knowledge of the general health of the patient, therefore collaboration with the attending physicians, the establishment of rigorous oral hygiene, personalized prophylaxis with a consequent contribution of fluorine.
\end{abstract}

Keywords: Alagille Syndrome; Oral Manifestations; Hypomineralization of the enamel; Oral Care

\section{Introduction}

Alagille syndrome is a rare autosomal dominant condition caused by mutations in the JAG1, NOTCH2, and other genes. The syndrome is characterized by cardiac malformations, facial abnormalities including micrognathia, short palpebral fissures, an upturned nose with anteverted nostrils and philtrum, short fingers with tapering tips and syndactyly of the second and third toes [1].

The symptoms of this genetic disease vary widely from person to person and may include congenital heart defects, difficulty swallowing, and liver problems.

Medical and dental management of Alagille syndrome is focused on preventing or treating heart problems, liver damage, jaundice due to birth defects in the bile ducts, and skeletal abnormalities.

\section{Case report}

The 6-year-old patient, accompanied by her mother, was referred to the Rabat Dental Diagnostic and Treatment Consultation Center (CCTD) (MOROCCO) for oral care.

She comes from a non-consanguineous marriage. The parents are in apparent good health. The mother also reports a great-grandmother who would have had the same syndrome. The pregnancy and childbirth went well with no special features. Alagille syndrome was diagnosed after three births, jaundice and genetic testing.

\footnotetext{
* Corresponding author: Adidémè Monique EZIN

University of Mohammed V, Faculty of dentistry,Departement of Pediatric Dentistry, Rabat-MOROCCO.

Copyright (C) 2022 Author(s) retain the copyright of this article. This article is published under the terms of the Creative Commons Attribution Liscense 4.0.
} 
The syndrome is associated in our patient with liver failure, a heart defect in the pulmonary and cardiovascular artery, an esophageal allergy and a gastric problem.

The patient is followed by medical staff: a hematologist, an endocrinologist, a gastrologist and a cardiologist.

The current medication consists of REFADINE, SODIUM BICARBONATE gel, URSOLVANT, VIT K, VIT E, VIT D, and growth hormones.

Hospitalization is done once a month for management of urinary infection and intermittent fever.

External oral examination of the patient reveals the presence of specific signs:

Pale skin tending to a yellowish appearance, a triangular face with a protruding forehead with a pointed chin. . (Fig 1, fig1')

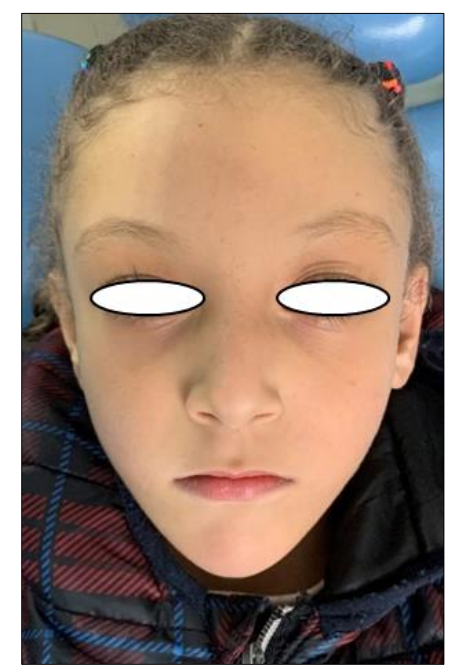

Figure 1 facial image visualizing the pale and yellow aspect of the skin (CCTD-RABAT-MOROCCO)

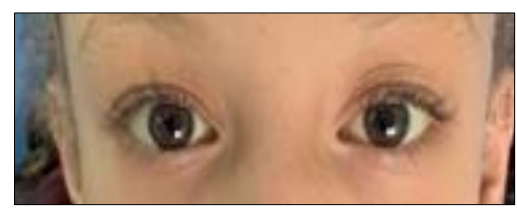

Figure 1 ' image of the yellow eyes (CCTD-RABAT-MO)

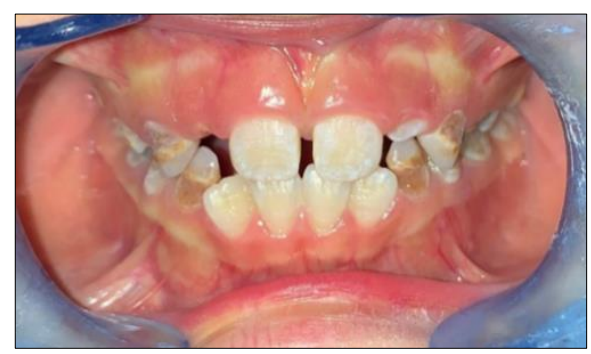

Figure 2 oral image visualizing the yellowish and pale aspect of the oral mucosa (CCTD-RABAT-MOROCCO)

The endo-oral examination revealed poor oral hygiene, a yellowish oral mucosa, and lips to the marginal gum. (Fig 2). There is also a whitish-looking tongue on its dorsal surface (geographic tongue) (fig 3), as well as a yellow palate (fig 4). 


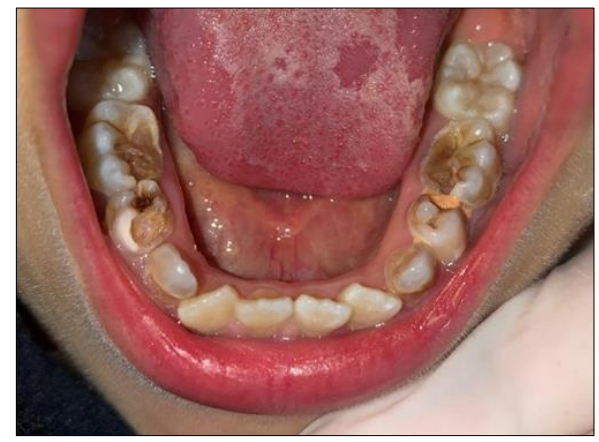

Figure 3 Oral image of the mandible visualizing the aspect of the tongue, the hypomineralization of the enamel and the carious lesions on teeth 73,74,75,83,84,85 (CCTD-RABAT-MOROCCO)

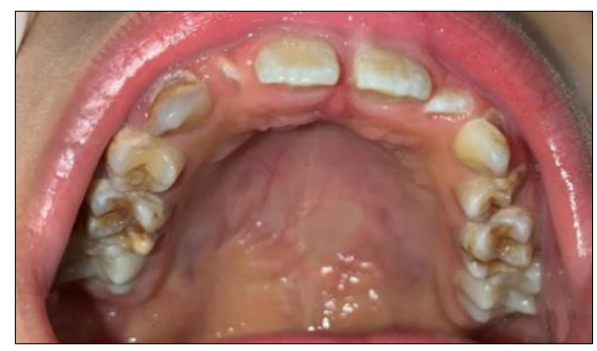

Figure 4 image buccale du maxillaire visualisant l'aspect du palais et l'immaturité de l'émail ainsi que les caries au niveau des dents 53,54,55,63,64,65 (CCTD-RABAT-MORROCO)

The examination of the teeth reveals a hypomineralization of the enamel of all the dentition (amelogenesis imperfect hypomature) both temporary and permanent, the presence of carious lesions of the canines and molars of the two maxillae. Advanced oral condition due to hypomineralization aggravated by poor hygiene and eating habits (fig3) (fig4).

The care of our patient was done in consultation with the multidisciplinary medical staff who treat this little girl. This pedodontic treatment consisted of prophylactic cleaning of the dental surfaces in order to reduce the oral bacterial load.

Due to the possible imperfect amelogenesis represented by the patient, means of individualized medium and long-term prophylaxis have been introduced: a rigorous motivation of oral hygiene, the prescription of a fluoridated toothpaste according to the latest recommendations (1500 ppm), periodic applications of fluorinated varnishes and ACP-CPP.

\section{Discussion}

Alagille syndrome is a rare genetic disorder that can affect the liver, eyes, heart and kidneys. It's a disease of the body's connective tissue, which helps support and protect various structures in the body. Connective tissues also help regulate cell growth and play important roles in blood clotting, wound healing and immunity.

A child with Alagille syndrome will have a narrow jawbone and distinctive facial features that include a pointed nose, full lips, and long eyelashes. In the first few months of life, babies with Alagille syndrome may have yellowing of the skin called jaundice.

Alagille syndrome is caused by a defective gene (by mutations in the JAG1, NOTCH2, and other genes) that controls the production of bile inside liver [2].

People with Alagille syndrome tend to have one of three problems: an enlargement in the size of their liver, a narrowing or total blockage of the bile ducts, or underdevelopment of their spleen and other parts of their immune system. This can make people with Alagille syndrome more vulnerable to infections. The enlarged livers are usually small enough that they do not cause pain or illness early on, but they may become large enough to cause problems later [3].

Oral manifestations of Alagille syndrome are common in children with the disease. Oral manifestations of Alagille syndrome can occur in up to $60 \%$ of patients. 
These Oral manifestations of Alagille syndrome include dry mouth, recurrent aphthous stomatitis dental fluorosis, lingual frenum hypertrophy, macroglossia, xerostomia, glossitis and an increased number of salivary glands. In addition, patients may present with a loss of taste sensation as well as dental anomalies such as open bite and malocclusion.

Other features that may be observed include enamel hypoplasia or amelogenesis imperfecta which leads to the formation of coarsely distributed fissures on the primary teeth surfaces (fig 3) (fig4).

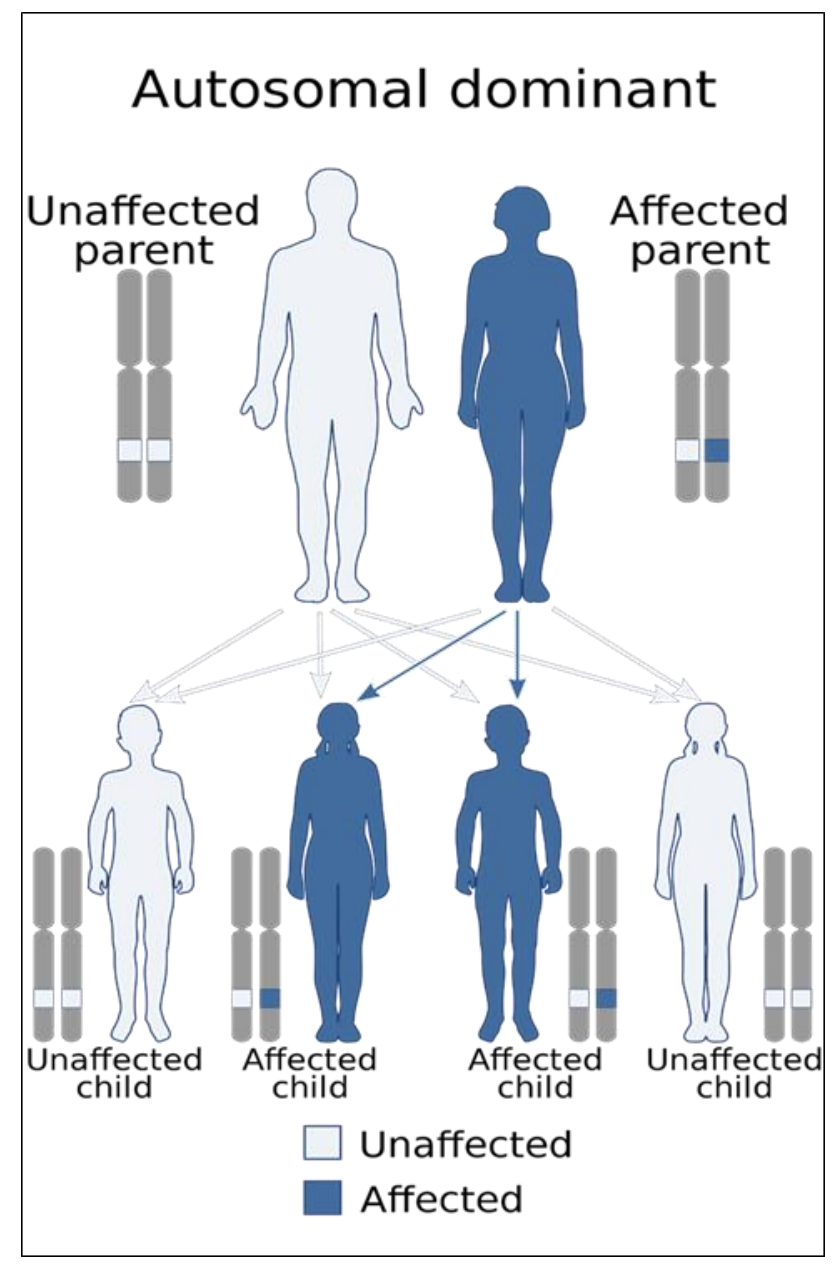

Figure 5 Genealogical tree of transmission of autosomal genes [6].

The medical and dental management of Alagille syndrome (AGS) is highly dependent on the type of disorder, the affected organ systems, and whether or not associated conditions are present [4].

The oral care of patients with a rare genetic disease such as AGS requires close collaboration with the entire medical stuff, but above all the establishment of a comprehensive treatment plan and a prevention strategy adapted to the patient.

It concerns an irreproachable oral hygiene, and a cari-resistant diet, the establishment of a rigorous follow-up.

The follow-up sessions will allow the early detection of cavities and their rapid dental management.

Adjusting oral conditions, along with eradicating infectious foci before liver transplantation, can reduce complications during medical procedures (fig3).

Oral hygiene instruction is an effective strategy for the reduction of pathogenic microorganisms. 
The role of the pedodontist is to become aware of the cardiovascular and hepatic problems associated with the syndrome in order to administer care that contributes to the overall well-being of the patient [5]. A pedodontist/pediatrician alliance to work in collaboration for better treatment is necessary.

\section{Conclusion}

Alagille syndrome (SGA)) is a rare genetic disorder, responsible for multiple liver, heart, skin, eye and spinal disorders. The cholestasis due to the illness leads to repercussions on the quality of the teeth structure and oral management.

Ambulatory oral management can be done in patients with AS, provided that the particularities of the syndrome are observed. The role of the dentist in maintaining oral health is important in order to avoid infectious foci, especially in immunocompromised patients. The dentist must also be aware of the cardiovascular and hepatic problems associated with the syndrome. Effective management contributes to the overall well-being of the patient. It is a permanent accompaniment.

\section{Compliance with ethical standards}

\section{Authors' contributions}

Adidémè Monique EZIN: Conceptualization, Writing original draft, \& editing. Rachid BOUDI: Writing, review \& editing. Hakima CHHOUL: Supervision, Validation, \& review.

\section{Disclosure of conflict of interest}

The authors declare that they have no conflicts of interest.

\section{Statement of informed consent}

The patient gave his informed consent to publish his case.

\section{References}

[1] Orpha.net: The portal for rare diseases AND orphan drugs. Accessed on. 10-24-21 at 14:30.

[2] Maha Saleh et Al, Alagille syndrome: clinical perpectives; the application of clinical genetics. 2016.

[3] Mirta Ciocca et Al, Alagille syndrome; Silver Bow Pediatrician. 2012.

[4] Hubert Désiré Mbassi Awa, et Al; Rare diseases AND their oral clinical manifestations in two hospital formations in Yaoundé, The Pan African Medical Journal. 2019.

[5] RIBEIRO, Eliane de Oliveira Aranha et Al, Features Dental approach in Alagille syndrome: Case report; Year 06, Ed.03, 16: 158-170 March 2021; 2448-0959, ISSN:2448-0959,SUFIXO DOI: 10.32749 Access Link: https: nucleodoconhecimento.com.br/dentistry/dental-approach.

[6] Medicine Portal en.wikipedidia.org, Genealogical tree of the transmission of autosomal genes. (accessed on 12 / 12/2021). 